\title{
Coproductive capacities: rethinking science-governance relations in a diverse world
}

Lorrae E. van Kerkhoff ${ }^{1}$ and Louis Lebel ${ }^{2}$

\begin{abstract}
Tackling major environmental change issues requires effective partnerships between science and governance, but relatively little work in this area has examined the diversity of settings from which such partnerships may, or may not, emerge. In this special feature we draw on experiences from around the world to demonstrate and investigate the consequences of diverse capacities and capabilities in bringing science and governance together. We propose the concept of coproductive capacities as a useful new lens through which to examine these relations. Coproductive capacity is "the combination of scientific resources and governance capability that shapes the extent to which a society, at various levels, can operationalize relationships between scientific and public, private, and civil society institutions and actors to effect scientifically-informed social change." This recasts the relationships between science and society from notions of "gaps" to notions of interconnectedness and interplay (coproduction); alongside the societal foundations that shape what is or is not possible in that dynamic connection (capacities). The articles in this special feature apply this concept to reveal social, political, and institutional conditions that both support and inhibit high-quality environmental governance as global issues are tackled in particular places. Across these articles we suggest that five themes emerge as important to understanding coproductive capacity: history, experience, and perceptions; quality of relationships (especially in suboptimal settings); disjunct across scales; power, interests, and legitimacy; and alternative pathways for environmental governance. Taking a coproductive capacities perspective can help us identify which interventions may best enable scientifically informed, but locally sensitive approaches to environmental governance.
\end{abstract}

Key Words: capacity development; coproduction; environmental governance; environmental policy; knowledge; scale; science-policy interface; sustainable development

\section{INTRODUCTION}

Effective responses to global environmental change demand effective relationships between science and governance (Bocking 2004, UNEP 2012, Cornell et al. 2013). Widespread concern over persistent inaction on pressing environmental issues has generated substantial interest in the complex relationships between science and policy, politics, and society (Cash et al. 2003, Clark and Dickson 2003, Jasanoff and Long Martello 2004, van Kerkhoff and Lebel 2006). Despite this ongoing research and scholarship, much of the analysis in this area has become embedded in the mantra of the "science-policy gap," and fails to address the diversity of challenges and opportunities that constructing and supporting these relationships entails (Kirchhoff et al. 2013, van Kerkhoff 2013). This metaphor is problematic because it implies a void waiting to be filled rather than a need to understand the coproduction landscape as a space already populated with formal and informal rules, practices, and capacities. An alternative perspective that has gained traction in recent years is that science and policy can be coproduced where the boundaries between these groups are blurred (Lemos and Morehouse 2005, Varady et al. 2013).

In this Special Feature we examine the concept of coproduction in relation to existing science-governance landscapes, particularly with regard to existing, and emerging, capacities. We draw on experiences from around the world to demonstrate and investigate the consequences of diverse capacities and capabilities in bringing science and governance together. We propose that coproductive capacities presents a new and useful concept through which to consider the diversity of contexts, challenges, and opportunities that shape the relations between science, governance, and implementation. This effectively brings together scholarship that recasts the relationships between science and society from notions of "gaps" to notions of interconnectedness and interplay (coproduction) alongside ideas that highlight societal foundations that shape what is or is not possible in that dynamic connection (capacities). The authors of the articles in this Special Feature explore this juxtaposition of coproductive capacity, to reveal social, political, and institutional conditions that both support and inhibit high-quality environmental governance as global issues are tackled in particular places. Importantly, they also demonstrate the different types of capacities that can be drawn upon to enable coproduction in diverse contexts, from environment and development economics in South Asia, to managing protected areas in Hawai'i. The coproductive capacity lens helps identify disparities and differences in sciencegovernance relations that lead to a range of environmental governance outcomes.

\section{COPRODUCTION}

There are two prevailing uses for the term "coproduction." The first and arguably original concept was developed by Jasanoff (2003, 2004), who described it as an idiom, stating that "the ways in which we seek to know the world both reflect and constitute the ways in which we choose to live in it" (Jasanoff 2004:1). In this view, science and governance interact not only with each other, but with social, cultural, and political norms. Jasanoff (2010:240) noted that "scientific facts bearing on the global environment never take root in a neutral interpretive field; they are dropped into contexts that have already been conditioned to produce distinctive cultural responses to scientific claims." The importance of recognising local contexts for co-production is also highlighted by Hulme (2010). He argues that prevailing discourses have globalized both knowledge making and decision making in relation to global environmental change concerns, rendering the 
diversity, richness, and challenges of local contexts largely invisible (Hulme 2010). This has generated schisms between universalized science focused on the production of global facts, and local meaning-making contexts in which such facts must be interpreted and acted upon (Jasanoff 2010). Both authors urge us to closely examine both decision making and knowledge making as dynamic and intimately connected processes across a range of socio-political scales.

The other use of coproduction refers to shared knowledge production at a project or program scale: "with experts and users 'co-producing' a shared body of usable knowledge" (Mitchell et al. 2004:109). This use of the term has gained acceptance in several circles such as the Future Earth global change research initiative, whose leaders argue that "a new kind of research, co-produced with society and seamlessly integrating social and natural sciences, is now needed to support a transition to global sustainability" (Future Earth 2012:2). Here coproduction emerges as an agenda, a call to configure and conduct our knowledge- and decision-making processes in particular ways, as yet poorly defined but implying homogeneity in that coproduction is not only needed, but essential "to support a transition to global sustainability." The return to universalizing language here ironically demonstrates, through the very use of the term coproduction, the schism referred to above, i.e., the desire for universal solutions, coupled with the recognition of the importance of rethinking how we approach and understand the relationships between science and governance at the project and program scale, in local contexts.

Clearly these two notions of coproduction are related. The first as a descriptive concept highlighting important relationships between science and governance that exist at multiple scales and are embedded in social, cultural, and political contexts; the second as an active, some might say prescriptive, normative concept whereby those relationships can and should be deliberately managed and enhanced for improving the scientific basis of decision making at the project and program scale. In considering these viewpoints, we want to avoid being drawn to one side or the other. We recognize the dangers of scientific determinism marginalizing alternative legitimate perspectives and knowledge in environmental governance processes; yet we also believe that science and research itself can be marginalized and excluded from decision-making contexts where it can make important contributions. Consequently, we seek to identify concepts and approaches that can draw fruitful connections between the universality of scientifically constructed global concerns, the ambiguity of bureaucratic power, and the necessary diversity and social embeddedness of localized responses.

\section{CAPACITY}

Capacity has long been established in literature and practice as a core issue in development across a range of international and domestic settings (UNDP 1998). Capacity refers broadly to "... the ability to perform functions, solve problems, and set and achieve objectives" (Fukuda-Parr et al. 2003:8) from societal to institutional and individual levels. Franks (1999) describes capacity as a combination of both capabilities required to act, and the competence to do so. The United Nations Development Program has proposed that capacity be considered on three levels: the enabling environment (broad system or context), the entity (or organization, institution), and the individual (UNDP 1998). More expansively, Eade (2007) describes capacities as individual, organizational, social, political, representational, material, technical, practical, and financial. Our idea of capacity in relation to coproduction and responses to global environmental change is concerned specifically with capacities to create, access, interpret, and apply scientific and research-based knowledge; and capacities to combine science with existing, localized knowledge, practices, and governance to effect change.

Capacity development is a related concept in development that has a wide range of interpretations and definitions. In its simplest terms, capacity development refers to the processes and interventions concerned with the intangible aspects of development, addressing the "increased ability to use and increase existing resources, in an efficient, effective, relevant and sustainable way" (Lavergne and Saxby 2001:4). Capacity development has become a core aim of development more broadly, reflecting a shift from more technical, top-down "knowledge transfer" approaches to more supportive, bottomup, endogenous development strategies. Virji et al. (2012) make a strong case for the importance of linking capacity development literature with global environmental change agendas. They argue that targeted investments to improve science capacities, as well as capacities to communicate and interact with policy makers are needed. In their view: "The need for this kind of capacity building is especially urgent in the developing world where adverse impacts from extreme climatic events and other manifestations of global change are compounded by poverty, environmental degradation, inadequate infrastructure, and weak governance" (Virji et al. 2012:115).

Bringing together concepts of coproduction and capacities as presented here implies an interest and concern for capacity development, as articulated by Virji et al. (2012). Our approach here, however, is more exploratory and descriptive than interventionist or evaluative. We believe it is important to first examine current capacities and challenges in coproduction across a diverse range of case studies. Importantly, we do not claim that these capacities determine particular abilities to bring about change; rather, they create the conditions under which effective, flexible, responsive governance can or cannot take place. Once the strengths and weaknesses of existing coproductive capacities are articulated, areas for support, intervention, and development may be identified and pursued.

\section{COPRODUCTIVE CAPACITY}

To do this we develop the concept of coproductive capacity, the foundations from which coproduction takes place. Coproductive capacity is the combination of scientific resources and governance capability that shapes the extent to which a society, at various levels, can operationalize relationships between scientific and public, private and civil society institutions and actors to effect scientifically informed social change. The concept of coproductive capacities draws from the original concept of coproduction to focus on those social, cultural, and political dimensions that explicitly and implicitly affect the relationships between knowledge making and decision making. It offers a conceptual base from which we can explore the diversity, variations, and disadvantages many societies face in seeking to respond to global environmental changes. It takes a normative 
position that processes of knowledge making and decision making and the relationships between them should be examined, understood, and supported at scales that are relevant to intervene in the problems at hand, countering the universalizing tendencies of global discourse and focusing on the dynamics of agency and action across a range of scales. It is also, however, instrumental, in the sense that it encourages the identification of both strengths and weaknesses in the scientific and governance resources available to tackle the complex tasks implied by environmental change, at those relevant scales.

Capacity and capacity development strongly resonate with coproduction in the context of environmental governance; they share a normative agenda of social transformation, and are grounded in belief in the transformative potential of new knowledge and learning. There are, however, also conflicts that emerge as coproduction and capacity are drawn together, particularly where technical or Western-style scientific-rational knowledge is implied to override local or customary knowledge, authority, and values. An important aspect of coproductive capacity as a concept is to reveal and examine the interplay and possible tensions between local and scientific knowledge, and how legitimacy and authority may be gained or lost in environmental decision making.

In this Special Feature we use the idea of coproductive capacity as a lens that seeks to differentiate the kinds of challenges confronted by different actors in various contexts in trying to address global environmental change. None of the projects reported on here were designed or conceptualized to enhance coproductive capacity; most were not even directly concerned with capacity development more broadly. What they do have in common, however, is that they were seeking to improve or understand the connections between scientific and research-based knowledge and governance of issues related to environment change. We include both successes and failures across the range of wealthy, middle-, and low-income countries, paying particular attention to those least-developed countries that are most vulnerable to global environmental change, and often have the fewest resources to respond. Where possible, authors pay special attention to minorities and marginalized communities, and the challenges confronted by those seeking to reconcile science-based management with alternative worldviews.

The authors of each of the papers presented here were asked to consider:

- What were the main governance challenges in the issues the project was addressing? How are they related to the knowledge resources needed or used?

- What were the main scientific and knowledge-based challenges? How are they related to governance capacities and priorities?

- What were the relationships between science and governance? How were these influenced by the context of the issue, and the conduct of the project?

\section{INSIGHTS FROM THE SPECIAL FEATURE}

The authors illustrate a range of lessons for developing the concept of coproductive capacity in theory and practice. We highlight themes that have emerged across several papers, and discuss their significance in relation to coproductive capacity.
History, experience, and preconceptions

Capacities for coproduction are historically and contextually embedded. History and experience are clearly important, shaping Jasanoff's (2010) "interpretive field" noted earlier in both positive and negative ways. Historical experiences are reflected in existing social and political institutions, either formally or informally. Schuttenberg and Guth (2015) point to the importance of existing relationships between researchers, Native Hawaiian cultural practitioners, and government that have been institutionalized through time. Previous efforts to bring these communities together had built a high level of capacity for engagement in the context of a new planning regime for a highly significant protected area. Direct experience of the area (site visits) also supported their ability to see and appreciate different social values in high-value conservation areas. Similarly, Wyborn (2015) notes that prior experience through the community-based Landcare movement in Australia created a context in which collaborative, cross-sectoral approaches to large-scale connectivity conservation made sense, but actors were unable to capitalize on this experience to realize effective coproduction. In contrast, her North American case study revealed that entrenched antiauthoritarian ideology in some locations prevented the conservation program from developing effective relationships with local landholders. Lebel et al. (2015) show that inexperience in evidence-based policy processes in Thailand shaped the issues that researchers needed to be able to engage with to construct positive relationships to support environmental governance. Part of the task implicit in their environmental assessments work was to build that experience and foster a culture that seeks to incorporate research-based evidence into decision making.

These authors show that social experiences and cultural preconceptions of science and government have historical roots, and that they can influence present day willingness to engage in coproduction processes. Efforts to develop or enhance coproductive capacities therefore need to identify and build from diverse existing relationships and experiences, paying close attention to the ways in which key actors and stakeholders formulate and reconcile the tensions between science and governance.

\section{Quality of relationships in suboptimal conditions}

The importance of understanding context is highlighted by the articles that demonstrate suboptimal conditions for coproduction. Suboptimal conditions draw our attention to the social, institutional, and political settings that appear to work against effective coproduction. All articles in this Special Feature demonstrate suboptimal conditions for coproduction to a greater or lesser extent; these are challenges that needed flexibility, innovation, and even risk-taking to overcome.

In Cambodia, Bowen et al. (2015) argue that although technical and financial support in the area of climate adaptation is needed, the creation of new organizational structures or investing in knowledge development will not yield results in conditions of mistrust and weak relationships. They stress that it is not just the presence of technical capacities in climate science or environmental governance that are necessary, but that they are connected in positive and trusting relationships. Efforts to develop coproductive capacities should take the fragility of this trust into account. Poor relationships and low trust can be a legacy of the past, but these legacies may emerge from forces external to the 
specific science-governance issues at hand; Mukhopadhyay et al. (2014) note that their South Asian research network operates across highly contested geopolitical boundaries of the IndoGangetic region. Under these conditions, rational discussions or action on global change issues that cross political borders are fraught with difficulty. The operation of their network creates a space in which such discussions may take place, enabling at least some consideration of regional environmental issues within their respective countries.

Recognizing where, how, and for whom conditions for coproduction are suboptimal is no small task. It may arise from the presence or absence of science or governance capacities, but also depends on the quality of the relationships between them. Building coproductive capacity requires processes of engagement that can support trust and commitment to improving sciencegovernance relationships, with the associated resources, time as well as funding, to do so.

\section{Disjunct across scales}

Science-governance relationships are multiscalar. Although governance structures such as projects and international agreements tend to direct our attention to particular scales of activity, coproduction in these papers did not fit neatly into a single scale of expertise, governance, or implementation. Many of the articles illustrate that successful coproductive relationships at one scale cannot assure, indeed, bear little relation at all to, success or failure to achieve the desired outcomes at other scales. As Wyborn (2015) shows, large-scale connectivity conservation groups can establish strong relationships between researchers and governing bodies, yet these can run into difficulties of implementation if local landholders have not been included effectively. This is not just a top-down challenge though. Comparing across Australia and North America, she shows that good capacities at the local scale in Australia struggled to "scale up" to the larger connectivity agenda implied by scientific discourse. Lebel et al. (2015) also note that different scales of governance have different perspectives on planning, reacting to existing environmental problems versus target-setting and visions for the future, and hence different expectations or needs from research projects.

Efforts to understand and facilitate coproductive capacity need to look across scales to identify important constraints or opportunities, where productive relationships at one scale cannot be assumed to translate to different scales of organization or action. Careful planning and consideration of scale in coproduction interventions has potential to broaden the reach of environmental governance projects, provided these scales are not assumed to align neatly.

\section{Power, interests, and legitimacy}

Science-governance relationships are shaped by power and interests. Although much suboptimality may be regarded as historical legacy, institutional lock-ins, or inattentiveness to important issues, the broader concept of coproduction serves as a reminder that these dimensions are produced and reproduced by relationships of power. Although power relations were an important part of scale politics (top down and bottom up) and suboptimality, they also had impacts horizontally, with implications for the legitimacy of coproduction activities. Bowen et al. (2015) note that the power exerted by international bodies to insist that Cambodia develop policy on climate change disregarded local (national and subnational) development priorities, which may explain lacklustre implementation of that policy. In this case the top-down imposition meets horizontal resistance at the local level.

In the development of ecosystem assessments in Thailand, Lebel et al. (2015) show that the interests of powerful actors shaped the boundaries of the assessments. Actors with capacity and political influence often successfully contested the scope, boundaries, and meaning of key terms in the assessments to enhance their own legitimacy in the assessment process. More positively, in locations where residents engaged governance actors and scientists simultaneously, those residents were empowered to inform and influence local planning and development. Even so, Lebel et al. note the challenges of including more marginalized communities through research. From this study we see that there is a delicate balance between fostering a culture of evidence-based policy making among the more powerful, while seeking to incorporate more marginalized local stakeholder needs, knowledge, interests, and values. Incorporating power and interests into consideration of how coproductive capacities may emerge or be constrained allows us to explore more subtle, locally relevant strategies for enhancing or developing those capacities.

\section{Alternative pathways for environmental governance}

Coproductive capacity can be actively shaped through interventions. The authors show that research projects, assessments, networks, environmental plans, or other initiatives can all build and enhance coproductive capacities. In our cases, these coproductive capacity-building outcomes were often incidental to specified project objectives. In some cases, considering projects or activities through a coproduction lens added an extra dimension to what was achieved. Schuttenberg and Guth (2015), for example, used coproductive capacity to articulate and define benefits and outcomes from their project that had not previously been captured. Mukhopadhyay et al. (2014) use the example of a major Indian Government report on greening national accounts that was developed in collaboration with a group of 13 high-level experts, $40 \%$ of whom had been supported by their network, to argue that policy gains are more likely to emerge from enhanced human capacity than from direct, project-based interventions. In other cases, coproductive capacity offered a way of critiquing existing projects and the relationships that developed within them. In this case the coproductive analysis is less about evaluating direct contributions to specific environmental governance outcomes, and more about how the project fostered the subtle growth of new, robust ways for decision makers to engage with environmental change through science and research.

Collectively, these cases illustrate that the contributions from investing in coproductive capacity may lie outside conventional project plans or project evaluation criteria. Incorporating coproductive capacity development explicitly into rules for funding or criteria for project monitoring and evaluation would create more systematic approaches that go beyond description and analysis to working out strategies, practical tools, and techniques. 


\section{CONCLUSION}

The variety of articles in this Special Feature has shown that the challenges and benefits of coproduction of science and governance are diverse, subtle, and nuanced across the spectrum of social settings. The authors of these articles demonstrate the value of critical examination of the emerging coproduction agenda, and the value of seeking to connect the more abstract concept of coproduction with localized capacities that may help or hinder effective environmental governance. Importantly, it moves us away from the homogenizing notion of science-policy gaps toward understanding the relationships between science and governance as contextualized, dynamic, and dependent on high quality relationships between actors both within and across scales. Indeed, despite the diversity of experiences and contexts illustrated in these articles, the key themes presented can start to offer guidance to those seeking to pursue coproduction as a practical agenda for improved environmental governance.

Although it is somewhat inevitable that we conclude this introductory paper with the comment that more research in this area is needed, we hope that the collection of articles in this Special Feature convince readers that although coproduction agendas hold great promise for advancing environmental governance, their complexity and subtlety should not be underestimated. Some priority research questions that emerge include:

- How can prior relationships between science and governance be understood and used to identify locally relevant pathways for building coproductive capacities?

- How can coproductive capacities be built in unfavorable contexts? Can they help overcome institutional or political constraints for better environmental governance?

- How does heterogeneity in governance capacities or power, scientific resources or knowledge, influence the prospects for coproductive capacity?

- How is coproductive capacity influenced by different levels of organization (project, program, institution)?

- How could coproductive capacity be incorporated into tangible (measurable) project goals or outcomes? How can we evaluate coproductive capacity interventions and continue to learn from experience in science-governance relationships?

The concept of coproductive capacities makes two contributions: one is a caution toward adopting new language and concepts without considering the underlying histories, contexts, and relationships from which the current relations between science and governance have emerged. The focus on capacities is intended to encourage researchers and practitioners to think about how coproduction is or is not embedded in existing capacities, from organizations and institutional structures through to power, interests, and the quality of relationships. Far from filling a gap, coproduction develops from this crowded and contested space. Considering existing coproductive capacities is one way to identify and analyze the complex contexts that support scientifically informed social change. The second contribution is that coproductive capacity can be fostered and enhanced, although perhaps a better metaphor is to suggest it is grown rather than implemented. From this perspective coproduction between science and governance emerges from positive interactions between actors and groups who have sufficient trust, willingness, and institutional room to manoeuvre to share information and decision-making power. The emphasis on capacities encourages us to consider coproduction in the context of the specific researchaction domains (places, topics, sectors) to which it is being applied. Attention to scale requires us to think not just in polarized top-down or bottom-up ways, but rather to draw attention to the connections within and between these scales and the ways actors can make those connections. Where interventions can focus on relationships and building cultures of trust in science and evidence, with sensitivity to local interests, knowledge, cultures, and institutions, capacities for coproduction may become self-sustaining. Continuing to share lessons around what works, what does not, and how to best support coproductive capacities as foundations for dealing with global environmental change is clearly an ongoing task.

The idea of coproduction holds substantial promise in relation to how we deal with the challenges of global environmental change. As highlighted by the UNEP Foresight Report, improving governance for sustainability is a top priority for the next century, and improving the connections between science and governance is central to this task (UNEP 2012). The Future Earth initiative's adoption of the language and concepts of coproduction to craft a positive vision of how these connections may unfold (Future Earth Transition Team 2013) testify to this, but to date there are few guidelines for how to enable and enact coproduction. Without such guidance we run the very real risk of returning to the determinism of traditional science-based approaches, with the tendency to work from the top down and privilege science over other forms of knowledge rather than integrating them. Coproduction is fundamentally a challenge to scientific elitism, and focusing on coproductive capacities is a way to better understand and accept that challenge in the diverse settings in which science can make a positive contribution to environmental governance.

Responses to this article can be read online at: http://www.ecologyandsociety.org/issues/responses. $\mathrm{php} / 7188$

\section{Acknowledgments:}

The authors would like to thank Carina Wyborn for comments on an earlier draft of this manuscript, and Boripat Lebel for editorial suggestions. We would also like to thank all contributing authors for their commitment to this special feature.

\section{LITERATURE CITED}

Bocking, S. 2004. Nature's experts: science, politics, and the environment. Rutgers University Press, Chapel Hill, North Carolina, USA.

Bowen, K. J., F. P. Miller, V. Dany, and S. Graham. 2015. The relevance of a coproductive capacity framework to climate change adaptation: investigating the health and water sectors in Cambodia. Ecology and Society 20(1): 13. http://dx.doi. org/10.5751/ES-06864-200113 
Cash, D. W., W. C. Clark, F. Alcock, N. M. Dickson, N. Eckley, D. H. Guston, J. Jäger, and R. B. Mitchell. 2003. Knowledge systems for sustainable development. Proceedings of the National Academy of Sciences of the United States of America 100 (14):8086-8091. http://dx.doi.org/10.1073/pnas.1231332100

Clark, W. C., and N. M. Dickson. 2003. Sustainability science: the emerging research program. Proceedings of the National Academy of Sciences of the United States of America 100 (14):8059-8061. http://dx.doi.org/10.1073/pnas.1231333100

Cornell, S., F. Berkhout, W. Tuinstra, J. D. Tàbara, J. Jäger, I. Chabay, B. de Wit, R. Langlais, D. Mills, P. Moll, I. M. Otto, A. Petersen, C. Pohl, and L. van Kerkhoff. 2013. Opening up knowledge systems for better responses to global environmental change. Environmental Science \& Policy 28:60-70. http://dx.doi. org/10.1016/j.envsci.2012.11.008

Eade, D. 2007. Capacity building: Who builds whose capacity? Development in Practice 17(4-5):630-639. http://dx.doi. org/10.1080/09614520701469807

Franks, T. 1999. Capacity building and institutional development: reflections on water. Public Administration and Development 19 (1):51-61. http://dx.doi.org/10.1002/(SICI)1099-162X(199902) 19:1<51::AID-PAD54>3.3.CO;2-E

Fukuda-Parr, S., L. Carlos, and M. Khalid. 2003. Capacity for development: new solutions to old problems. Earthscan, London, UK.

Future Earth. 2012. Future Earth: research for global sustainability: a framework document. Future Earth, International Council for Science, Paris, France. [online] URL: http://www.icsu. org/future-earth/media-centre/relevant_publications/future-earthframework-document

Future Earth Transition Team. 2013. Future Earth initial design. Future Earth, International Council for Science, Paris, France. [online] URL: http://www.icsu.org/future-earth/media-centre/ relevant publications/future-earth-initial-design-report

Hulme, M. 2010. Problems with making and governing global kinds of knowledge. Global Environmental Change 20:558-564. http://dx.doi.org/10.1016/j.gloenvcha.2010.07.005

Jasanoff, S. 2003. Breaking the waves in science studies: comment on H. M. Collins and Robert Evans, 'The Third Wave of Science Studies.' Social Studies of Science 33:389-400. http://dx.doi. org/10.1177/03063127030333004

Jasanoff, S. 2004. States of knowledge: the co-production of science and social order. Routledge, London, UK. http://dx.doi. org/10.4324/9780203413845

Jasanoff, S. 2010. A new climate for society. Theory, Culture and Society 27:233-253. http://dx.doi.org/10.1177/0263276409361497

Jasanoff, S., and M. Long Martello. 2004. Earthly politics: local and global in environmental governance. MIT Press, Cambridge, Massachusetts, USA.

Kirchhoff, C. J., M. C. Lemos, and S. Dessai. 2013. Actionable knowledge for environmental decision making: broadening the usability of climate science. Annual Review of Environment and Resources 38:393-414. http://dx.doi.org/10.1146/annurevenviron-022112-112828
Lavergne, R., and J. Saxby. 2001. Capacity development: vision and implications. Capacity Development Occasional Series, Vol. 6088:0-11. Canadian International Development Agency, Gatineau, Québec, Canada.

Lebel, L., S. Wattana, and P. Talerngsri. 2015. Assessments of ecosystem services and human well-being in Thailand build and create demand for coproductive capacity. Ecology and Society 20 (1): 12. http://dx.doi.org/10.5751/ES-06527-200112

Lemos, M. C., and B. J. Morehouse. 2005. The co-production of science and policy in integrated climate assessments. Global Environmental Change 15(1):57-68. http://dx.doi.org/10.1016/j. gloenvcha.2004.09.004

Mitchell, R. B., W. C. Clark, D. W. Cash, and F. Alcock. 2004. Science, scientists, and the policy process: lessons from global environmental assessments for the northwest forest. Pages 95-111 in K. Arabas and J. Bowersox, editors. Forest futures: science, politics and policy for the next century. Rowman and Littlefield, Lanham, Maryland, USA.

Mukhopadhyay, P., M. Nepal, and P. Shyamsundar. 2014. Building skills for sustainability: a role for regional research networks. Ecology and Society 19(4): 45. http://dx.doi. org/10.5751/ES-07105-190445

Schuttenberg, H. Z., and H. K. Guth. 2015. Seeking our shared wisdom: a framework for understanding knowledge coproduction and coproductive capacities. Ecology and Society, in press.

United Nations Development Programme (UNDP). 1998. Capacity assessment and development in a systems and strategic management context. UNDP. New York, New York, USA.

United Nations Environment Programme (UNEP). 2012. 21 Issues for the 21 Century: results of the UNEP foresight process on emerging environmental issues. UNEP, Nairobi, Kenya. [online] URL: http://www.unep.org/pdf/Foresight Report-21 Issues fo$\underline{r}$ the 21st Century.pdf

van Kerkhoff, L. 2013. Knowledge governance for sustainable development: a review. Challenges in Sustainability 1(2):82-93. http://dx.doi.org/10.12924/cis2013.01020082

van Kerkhoff, L., and L. Lebel. 2006. Linking knowledge and action for sustainable development. Annual Review of Environment and Resources 31:445-477. http://dx.doi.org/10.1146/ annurev.energy.31.102405.170850

Varady, R. G., C. A. Scott, M. Wilder, B. Morehouse, N. P. Pablos, and G. M. Garfin. 2013. Transboundary adaptive management to reduce climate-change vulnerability in the western U.S.-Mexico border region. Environmental Science \& Policy 26:102-112. http:// dx.doi.org/10.1016/j.envsci.2012.07.006

Virji, H., J. Padgham, and C. Seipt. 2012. Capacity building to support knowledge systems for resilient developmentapproaches, actions, and needs. Current Opinion in Environmental Sustainability 4:115-121. http://dx.doi.org/10.1016/j.cosust.2012.01.005

Wyborn, C. A. 2015. Connecting knowledge with action through coproductive capacities: adaptive governance and connectivity conservation. Ecology and Society 20(1): 11. http://dx.doi. org/10.5751/ES-06510-200111 